\title{
Control of mRNA decapping by positive and negative regulatory elements in the Dcp2 C-terminal domain
}

\author{
FENG HE and ALLAN JACOBSON \\ Department of Microbiology and Physiological Systems, University of Massachusetts Medical School, Worcester, Massachusetts 01655, USA
}

\begin{abstract}
Decapping commits an mRNA to complete degradation and promotes general $5^{\prime}$ to $3^{\prime}$ decay, nonsense-mediated decay (NMD), and transcript-specific degradation. In Saccharomyces cerevisiae, a single decapping enzyme composed of a regulatory subunit (Dcp1) and a catalytic subunit (Dcp2) targets thousands of distinct substrate mRNAs. However, the mechanisms controlling this enzyme's in vivo activity and substrate specificity remain elusive. Here, using a genetic approach, we show that the large C-terminal domain of Dcp2 includes a set of conserved negative and positive regulatory elements. A single negative element inhibits enzymatic activity and controls the downstream functions of several positive elements. The positive elements recruit the specific decapping activators Edc3, Pat1, and Upf1 to form distinct decapping complexes and control the enzyme's substrate specificity and final activation. Our results reveal unforeseen regulatory mechanisms that control decapping enzyme activity and function in vivo, and define roles for several decapping activators in the regulation of mRNA decapping.
\end{abstract}

Keywords: mRNA decapping; decapping enzyme; decapping activators; positive and negative regulation

\section{INTRODUCTION}

Decapping plays an important role in multiple aspects of eukaryotic cytoplasmic mRNA turnover (Song et al. 2010; Parker 2012) and is critical to numerous biological processes, including development (Xu et al. 2006; Ma et al. 2013), DNA replication (Mullen and Marzluff 2008; Schmidt et al. 2011), stress response (Hilgers et al. 2006; Xu and Chua 2012), synapse plasticity (Hillebrand et al. 2010), retrotransposition (Dutko et al. 2010), and viral replication (Hopkins et al. 2013). In yeast, mRNA decapping is carried out by a single enzyme composed of a regulatory subunit (Dcp1) and a catalytic subunit (Dcp2), but also requires the functions of specific regulators commonly designated as "decapping activators" (Parker 2012). Decapping of general mRNAs requires the functions of the decapping activators Pat1, Dhh1, Edc3, and the Lsm1-7 complex, and decapping of nonsense-containing mRNAs requires the NMD-specific regulators Upf1, Upf2, and Upf3. All these decapping factors are conserved from yeast to humans, but their precise functions and modes of regulation, and that of the decapping enzyme itself, are largely unknown. There are, however, mechanistic models for decapping enzyme regulation and these models are largely based on the results of in vitro enzymatic assays (Steiger et al. 2003; Harigaya et al. 2010; Borja et al. 2011), structural anal-

Corresponding authors: feng.he@umassmed.edu; allan.jacobson@ umassmed.edu

Article published online ahead of print. Article and publication date are at http://www.rnajournal.org/cgi/doi/10.1261/rna.052449.115. yses (She et al. 2006, 2008; Deshmukh et al. 2008; Floor et al. 2010), and protein-protein interaction studies (Decker et al. 2007; Nissan et al. 2010). These experiments have led to the notion that the catalytic step is limiting for mRNA decapping and that decapping activators function mainly to enhance decapping chemistry, possibly by promoting a conformational change in Dcp2 required for the decapping reaction (Floor et al. 2010; Borja et al. 2011). However, essentially all of the studies on which the mechanistic models are based have two significant shortcomings. First, they used Dcp2 fragments lacking approximately $700 \mathrm{C}$-terminal amino acids of that protein's 970 residues. Although the large C-terminal truncation of Dcp2 yielded an apparently functional decapping enzyme (Dunckley and Parker 1999), the resulting Dcp2 fragment could lack important regulatory activities. And, second, the in vitro decapping experiments used generic mRNAs as substrates, and thus missed the opportunity to determine how the decapping enzyme controls its target specificity critical for in vivo regulation.

Here, we investigate the molecular mechanisms involved in regulating the yeast decapping enzyme in vivo. Using deletions, point mutations, and two-hybrid analysis to dissect protein:protein interactions, and quantitative Northern analyses of multiple mRNAs to assess mRNA decay activity, we

(C) $2015 \mathrm{He}$ and Jacobson This article is distributed exclusively by the RNA Society for the first 12 months after the full-issue publication date (see http:// rnajournal.cshlp.org/site/misc/terms.xhtml). After 12 months, it is available under a Creative Commons License (Attribution-NonCommercial 4.0 International), as described at http://creativecommons.org/licenses/by-nc/4.0/. 
identified the decapping activators that bind to Dcp2 and revealed that the yeast decapping enzyme is subject to both negative and positive regulation. Surprisingly, the key regulatory elements for both negative and positive regulation all mapped to the C-terminal domain of Dcp2 that had been neglected in almost all previous in vitro decapping experiments.

\section{RESULTS}

\section{A small subset of mRNA decapping activators interacts with the Dcp2 catalytic subunit of the yeast decapping enzyme}

To delineate the molecular mechanisms involved in decapping enzyme regulation, we first identified the factors that interact with Dcp1 and Dcp2. Using the yeast two-hybrid assay (Fields and Song 1989), we tested for potential interactions between Dcp1 or Dcp2 and the general decapping activators Edc3, Dhh1, Pat1, and Lsm1, or the NMD-specific regulators Upf1, Upf2, and Upf3. In parallel, and as a positive control, we also analyzed interactions between Dcp1 and Dcp2. In this initial set of experiments, we analyzed full-length Dcp1 and Dcp2, as well as a Dcp2 C-terminal fragment lacking the protein's conserved N-terminal 244-amino acid catalytic domain. We included the Dcp2 C-terminal domain in our initial experiments because Dcp2 may exhibit conformation-dependent interactions with its interacting partners. The interactions of Dcp1 or Dcp2 with the decapping activators were tested in both Gal4-DB and Gal4-AD orientations and success in either orientation was deemed evidence for interaction. As shown in Supplemental Figure S1A, Dcp1 and Dcp2 interacted with each other as expected. However, the interactions of Dcp 1 and Dcp2 with the decapping activators were more complex. When tested against the full-length proteins, Edc3 interacted with both Dcp1 and Dcp2, Dhh1 and Upf1 interacted with Dcp1 but not Dcp2, and Pat1, Lsm1, Upf2, and Upf3 failed to interact with either Dcp1 or Dcp2. However, both Pat1 and Upf1 interacted strongly with the C-terminal Dcp2-ND244 fragment lacking the protein's catalytic domain. The lack of positive two-hybrid interactions of Lsm1, Upf2, and Upf3 with either subunit of the decapping enzyme was not likely to be due to insufficient expression or misfolding of the respective Gal4-DB or AD fusion proteins in yeast cells as each of these fusion proteins interacted with their respective positive controls (Supplemental Fig. S2A and data not shown).

Since protein:protein interaction results can reflect "bridging" by interacting partners, we generated tester strains harboring complete deletions of EDC3, PAT1, DHH1, and UPF1, or a C-terminal deletion of DCP2 (N245 allele) (He et al. 2014), and assayed each binary interaction in these strains. The N245 allele lacks Dcp2's C-terminal 725 amino acids, including its Edc3-binding site (Fig. 1A; Harigaya et al. 2010), and was utilized because DCP1 and DCP2 were essential and could not be deleted completely in our tester strain. As shown in Supplemental Figure S1B, the interactions between Dcp2 and Dcp1, Edc3, Pat1, or Upf1 still occurred in each deletion/truncation strain. However, interactions between Dcp1 and Edc3 or Upf1 were largely eliminated in the $d c p 2-N 245$ strain, as were interactions between Dcp 1 and Dhh1 in both the $d c p 2-N 245$ and edc3s strains. Dhh1 interacted with Edc3 in all the tester strains (Supplemental Fig. S2B), suggesting direct interactions between these two factors, as recently demonstrated by in vitro experiments (Sharif et al. 2013). Together, these results indicate that the decapping enzyme interacted directly with only three decapping activators, Edc3, Pat1, and Upf1, and that all three interactions are mediated by Dcp2. Further, the use of tester strains harboring specific deletions or truncations allowed us to conclude that the observed interactions between Dcp1 and Edc3 or Upf1 were bridged through Dcp2 and that between Dcp1 and Dhh1 was bridged through both Dcp2 and Edc3.

These two-hybrid data validate previously observed in vitro interactions between Dcp2 and Edc3 (Harigaya et al. 2010), but challenge other results derived from in vitro pull-down experiments, including those suggesting direct interactions between Dcp1 and Pat1 (Nissan et al. 2010) or between Dcp2 and Dhh1 (Decker et al. 2007). Previous in vitro pull-down experiments also revealed a direct interaction between Dcp2 and Pat1 (Nissan et al. 2010), but this Dcp2:Pat1 interaction differed from that shown by our two-hybrid assays. The in vitro Dcp2:Pat1 interaction was mediated by the N-terminal 300-amino acid fragment of Dcp2 whereas our two-hybrid Dcp2:Pat1 interaction was mediated by a nonoverlapping C-terminal fragment of Dcp2 (see below).

\section{Discrete and generally nonoverlapping Dcp1-, Edc3-, Pat1-, and Upf1-interacting domains within Dcp2}

To elucidate the potential roles of Dcp1, Edc3, Pat1, and Upf1 in regulating Dcp2 activity, we used the two-hybrid assay and systematic DCP2 deletions to map the binding sites of each factor on Dcp2 (Fig. 1A,B). Consistent with previous biochemical analyses (She et al. 2008; Harigaya et al. 2010), Dcpl bound to an N-terminal region encompassing the first 100 amino acids of Dcp2, and Edc3 bound to an internal region (amino acids 246-300) immediately downstream from the Dcp2 catalytic Nudix domain. Pat1 and Upf1 interacted with overlapping or neighboring Dcp2 C-terminal domain fragments. However, the actual Dcp2 binding sites for these two factors appeared to be distinct: Pat1 bound to five small segments from three different regions (amino acids 416-525, 721-880, and 881-970) (Fig. 1A) and Upf1 bound strongly to two small segments (amino acids 416-475 and 661-720), but failed to interact with the 300-amino acid N-terminal Dcp2 segment observed in a previous two-hybrid analysis (Fig. 1B; Swisher and Parker 2011). The Pat1-interacting segments do not exhibit significant overall sequence similarity, but each shares at least one short 5-amino acid leucine-rich 
A

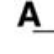

A

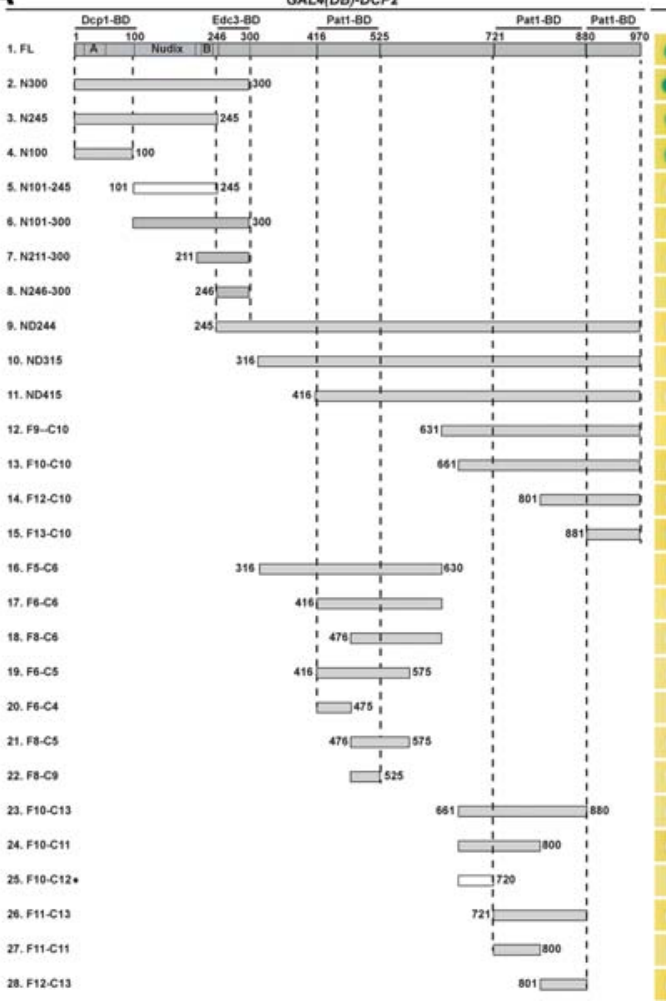

GALA(AD)

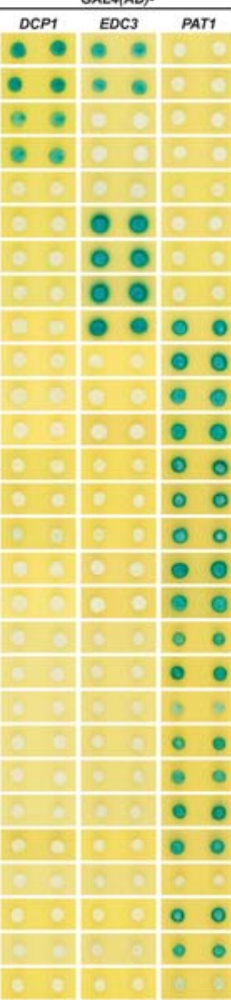

B

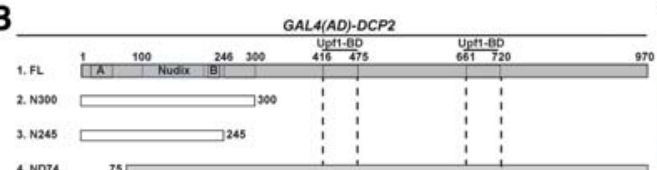

4. NO74

S. NO2e4

a. Nos15

7. . Fsece

8. Fs-CS

9. Fs:C4

10. Fs.ca

11. Fo.

12. Fo-Cs

13. F6.CA

14. Fa.C6.

13. Fo.cs.

16. Facco.

17. F9-C10

18. F10.c10

19. F11.610

20. F12.c10.

21. F13.C10.

22. F10.-13

23. F10-c11

24. F10.-12

25. F11.C11.

26. F11.C13.

27. F12-c13.
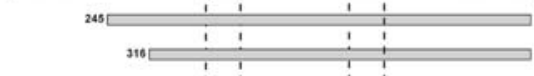

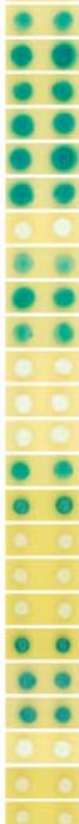

FIGURE 1. Identification of Dcp1, Edc3, Pat1, and Upf1-interacting domains of Dcp2. (A) Mapping of Dcp1, Edc3, and Pat1-interacting domains of Dcp2. (B) Mapping of Upf1-interacting domains of Dcp2. Dcp2 fragments fused to Gal4(DB) or Gal4(AD) were assayed for interactions with Gal4 $(\mathrm{AD})-\mathrm{Dcp} 1, \mathrm{Edc} 3$, and Pat1 $(A)$ or Gal4(DB)-Upf1 $(B)$, respectively. $A$ and $B$ include schematic representations of the Dcp2 domain structure and the Dcp2 fragments used in the experiment. Shaded bars indicate interacting fragments, whereas white bars indicate fragments that failed to interact. The minimal binding sites for Dcp1, Edc3, Pat1, and Upf1 are bracketed by dashed lines. In $A$ and $B$, blue colony color indicates an interaction and white colony color indicates no interaction. Constructs highlighted with a black diamond indicate Dcp2 segments that interact with Upf1, but not Pat1, or vice versa.

motif, suggesting that these motifs are directly involved in Pat1 binding. A total of eight such motifs were identified in these Pat1-interacting segments (Fig. 2A). Multiple leucinerich motifs were also identified in the C-terminal domain of S. pombe Dcp2 (Fromm et al. 2012). In contrast to our two-hybrid data, previous in vitro pull-down and NMR analyses indicated that some of these motifs bind Edc3 or Scd6 (Fromm et al. 2012). The two Upf1-interacting segments exhibit significant similarity over their entire 60 -amino acid regions (data not shown), but also share extremely high local identity over an 11 amino acid stretch (eight out of 11) (Fig. 2A). The Dcp1, Edc3, Pat1, and Upf1-interacting domains or motifs identified here likely play important regulatory roles, as each of these domains or motifs is conserved during eukaryotic evolution and exists in the vast majority of fungal Dcp2 orthologs (Fig. 2A; Supplemental Fig. S3). Short linear interacting motifs have also been identified in many other decapping factors from yeast to humans and appear to be localized primarily to the disordered regions of these proteins (Jonas and Izaurralde 2013), suggesting that protein-protein interactions mediated by short linear motifs may be a general feature of eukaryotic decapping regulators.

\section{Mapping of Dcp2-interacting domains of Edc3, Pat1, and Upf1}

Using similar assays, we also identified the domains of Edc3, Pat1, and Upf1 involved in Dcp2 binding. Since both Edc3 and Upf1 can self-associate (Decker et al. 2007; He et al. 2013), we utilized two-hybrid tester strains with and without complete deletions of EDC3 or UPF1. Full-length Edc3 interacted strongly with Dcp 2 in $e d c 3 \Delta$ cells and this interaction was eliminated by deletion of Edc3's Lsm domain and substantially diminished by deletion of the YjeF-N domain (Supplemental Fig. S4A). These data suggest that Dcp2 binding by the Edc3 Lsm domain is weak and requires enhancement by the YjeF-N dimerization domain. Consistent with this interpretation, Edc3 fragments containing the YjeF-N domain but lacking the Lsm domain showed no interaction with Dcp2 in edc3 $\Delta$ cells, but exhibited fairly strong interaction with Dcp2 in wild-type cells (Supplemental Fig. S4A, construct F2-C1). Dcp2 interacted strongly with the Cterminal domain of Pat1 (amino acids 422 to 796) (Supplemental Fig. S4B, construct F3-C1), a region that forms repeat helical structures and is also involved in binding the Lsm1-7 
A

Edc3-binding motif
S. cerevisiae
S. paradoxus
S. mikatae
S. bayanus
C. glabrata
S. castellii
K. waltii
S. kluyveri
C. Iusitaniae
D. hansenii
C. guilliermondii
C. tropicalis
C. albicans
C. parapsilosis
Consensus ( $80 \%$ )
(246) QLKSYAEEQLKLLLGIT---KEEQIDP GRELLNMLHTAVQ (282)
QLKSYAEEQLKLLLGIT---KEEQIDPGRELLNMLHTAVQ
QLKSYAEEQLKLLLGIT---KEEQIDPGRELLNMLHTAVQ
QLKTYAEEQLKLLLGIT---KEEQIDPGRELLNMLHTAVQ
QSKKYAEEQLKLMLGIT---KEENID PGRELLNMLQSSVH
QLKQYAEEQLKLLLGIT---KGEQVDPGRELLNMLHSAVH
KLKSHVEAQLKLLLGIR---KEETPD PGRELLNMLQNSVT
KLKLYAEEQLKLLLGIK---KDETVDPGRELLNMLQTAVT
QLKRDAEVRLKALMGISD-VVSENTDAGRELLDLLQGAAK ELMLQAE IKLKSLLGISQP-KLENLDAGRELLNILQGVIP ELLVKAEFRLKEYLGISP-KAQENVDAGRELLNILQGSSK E IMLQVELKLKDLLGLN--KPVENVDAGRELLN ILQKVGQ E IMLQVELKLKELLGLNAKEPEENVDAGRELLNILQKVGQ ELMQQAEIKLKKLMGLN--KCEENVDAGRELLNILQRAKP
plh..sE.pLK.LLGls. .htEplDsGRELLNhLptssp

Upf1-binding motifs

S. cerevisiae
S. paradoxus
S. mikatae
S. bayanus
C. glabrata
K. waltii
S. kluyveri
S. cerevisiae
S. paradoxus
S. mikatae
S. bayanus
C. glabrata
S. castellii
K. waltii
S. kluyveri
C. lusitaniae
D. hansenii
C. guiliermondii
C. tropicalis
C. albicans
C. parapsilosis

(456) VQSSKKPKLKILQRGTDLNS (475) VQSNKKPKLKILQRGTDLNS VQSSNKPKLKILQRGTDLNS EQISEKPKLKILQRGTDLNS ETITAKPKIKILKRGENLSL PQESTKPKFTILKRGQNLEN KDDEKKPKLKLLKRGQTLQD

(694) NTVKKVPKVKILKRGETFAS (713) NTVKKTPKVKILKRGETFAS NTIKKMPKVKILKRGETFAS STIKKTPKVKLLKRGETIAT QVAGPKPKVKILKRGEILNS KENVKKPKFKILKRGENISD SNVKQKPKFKLLKRGESLRD SNPQVRPKIKLLKRGQKLGD ASKTDGPKITLLKRAPNEDA NRKEAGKKLTLLKRPTDAGS RPSTPAGKLKILKRPTASGD QPVGAGKKIKLLKRSDNKAS VPVTSRRKIKLLKRSDNEAS RQPVVGQKIKLLKRSDNODS .p.pttsKIKILKRupshss

\section{Pat1-binding motifs}

Pat1-BD-1
Pat1-BD-2
Pat1-BD-3A
Pat1-BD-3B
Pat1-DB-4
Pat1-DB-5A
Pat1-DB-5B
Pat1-DB-5C

S. paradoxus

S. mikatae

S. bayanus

C. glabrata

S. castellii

K. waltii

S. kluyveri

C. Iusitaniae

C. tropicalis

C. albicans

C. parapsilosis

D. hansenii

C. guilliermondii Consensus ( $80 \%$ )
(440) PGQLLDILNSK (450)

(489) SQALLDLLKKP (499)

(730) SKDLLQMLRNP (740)

(792) ASELLGMLKQK (802)

(824) AKGLLNILKKN (834)

(894) SHELLNILHGN ( 904$)$

(931) SNKLLNMLQNR ( 941 )

(960) SNELLSILHRK (970)

\begin{abstract}
PGOLLDLLNSK
PGQLLNLLHSK SROLLNLLNSK SKELLNLLRSK GKQLLDILHSN SKOLLDLLNRK SKELLDLLKKK SKELISILKGG SKELLSILTTK SKELLSILTAK SKELLSILTTK SKEFLSILNKP SKEFLSILSOP uppLIslLppt
\end{abstract}

B

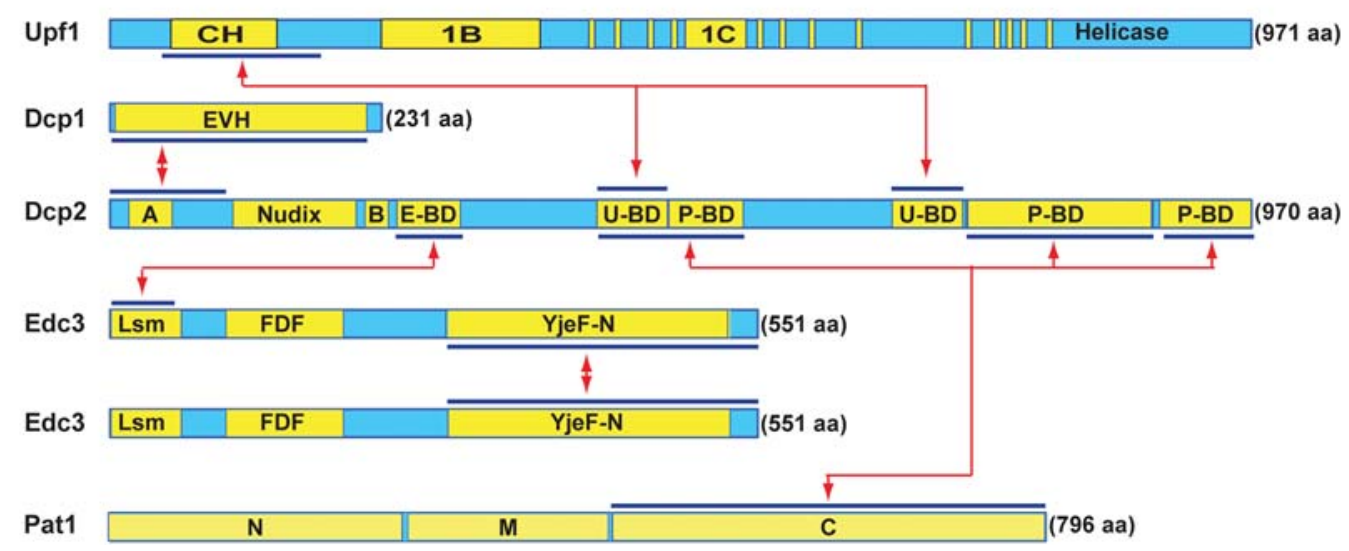

FIGURE 2. Identification of the conserved Edc3, Pat1, and Upf1-binding Motifs in the C-terminal domain of Dcp2 and a summary of the interacting domains involving Dcp2 and Dcp1, Edc3, Pat1, and Upf1. (A) Multiple sequence alignments of Dcp2's Edc3-, Upf1-, and Pat1-binding motifs with the respective conserved sequences from other fungal Dcp2 orthologs. Amino acids are colored based on physicochemical properties ([h] hydrophobic, [l] aliphatic, [p] polar, [s] small, [t] turn-like, $[\mathrm{u}]$ tiny). (B) Protein domains involved in interactions between Dcp1 and Dcp2, and between Dcp2 and Edc3, Pat1, or Upf1. Schematic representations of the domain structures for each factor are shown. Interactions between factors are indicated by red lines with arrowheads, and domains implicated in each interaction are marked by straight blue lines. For Dcp2, E-BD indicates Edc3-binding domain, U-BD indicates Upf1-binding domain, and P-BD indicates Pat1-binding domain. 
complex (Sharif and Conti 2013; Wu et al. 2014). Binding of Dcp2 to Upf1 was found to be mediated by amino acids 62181 of the Upf1 CH-domain (Supplemental Fig. S4C, construct 2-16). In contrast to a previous study (Swisher and Parker 2011), we found that deleting UPF1, EDC3, or PAT1 from the tester strain did not affect Dcp2 binding to this Upf1 region (Supplemental Fig. S4C), indicating that the observed Dcp2:Upf1 interaction is not bridged by any of these factors. This region of Upf1 is also involved in Upf2 binding (He et al. 1997) and self-association (He et al. 2013), suggesting that it may play a role in sequential molecular interactions during activation of NMD.

\section{Dcp2 C-terminal domain contains an inhibitory element that subjects the enzyme to negative regulation}

Even though our two-hybrid analyses identified specific Dcp2 C-terminal domain binding sites for Edc3, Pat1, and Upf1 (Fig. 2B), it was important to determine whether these sites had functional significance. Accordingly, we evaluated their possible regulatory roles by generating incremental Dcp2 C-terminal deletions and analyzing their consequences on the steady-state levels of YRA1 pre-mRNA in the presence or absence of Edc3, i.e., in $d c p 2 \Delta$ or edc3 $3 d c p 2 \Delta$ cells. YRA1 pre-mRNA is a substrate of the Edc3-mediated decay pathway and we have previously shown that this transcript is degraded in the cytoplasm by a translation-independent decapping mechanism (Dong et al. 2010). DCP2 wild-type and deletion mutant alleles on centromere-based plasmids were individually introduced into $d c p 2 \Delta$ and $e d c 3 \Delta d c p 2 \Delta$ cells and YRA1 pre-mRNA levels in the resulting cells were analyzed by Northern blotting. Cells harboring the empty vector served as negative controls. In the presence of Edc3 (i.e., in $d c p 2 \Delta$ cells), Dcp2 C-terminal deletions up to amino acid 245, including the Edc3-binding site, had no apparent consequence on YRA1 pre-mRNA decay. Almost all the mutant alleles behaved like the wild-type allele and promoted efficient YRA1 pre-mRNA degradation (Fig. 3A). Cells harboring the $d c p 2-\mathrm{N} 300$ and $\mathrm{N} 245$ alleles had subtle differences from the other alleles, consistently accumulating slightly lower or higher levels of YRA1 pre-mRNA, respectively (Fig. 3A). The $d c p 2-N 210$ allele, whose large deletion encompasses box B sequences of the Nudix domain, appeared to eliminate Dcp2 function for YRA1 pre-mRNA. Cells harboring the $d c p 2-N 210$ allele were also defective in NMD and general mRNA decay (data not shown), indicating that this allele encodes a nonfunctional decapping enzyme. In the absence of Edc3 (i.e., in $e d c 3 \Delta d c p 2 \Delta$ cells), the $d c p 2$ alleles that were apparently functional in $d c p 2 \Delta$ cells yielded two distinct decay phenotypes. Alleles harboring deletions up to amino acid 475 behaved like wild-type $D C P 2$, i.e., edc3 $3 d c p 2 \Delta$ cells harboring these alleles accumulated high levels of YRA1 pre-mRNA (Fig. 3A, lanes 2-5), indicating a failure to promote YRA1 pre-mRNA decapping in the absence of
Edc3 activation. In contrast, $e d c 3 \Delta d c p 2 \Delta$ cells with $d c p 2$ alleles harboring deletions up to amino acids 300 or $245 \mathrm{ac}-$ cumulated significantly lower levels of YRA1 pre-mRNA (Fig. 3A, lanes 6,7), indicating an ability to promote YRA1 pre-mRNA decapping even in the absence of Edc3. These results indicate that the Dcp2 C-terminal domain contains a cis-inhibitory element between amino acids 300-475, and that this element inhibits YRA1 pre-mRNA decay in the absence of Edc3.

To determine whether the decreased steady-state accumulation of YRA1 pre-mRNA in $e d c 3 \Delta d c p 2 \Delta$ cells harboring the $d c p 2-N 300$ or N245 allele is directly attributable to increased decapping activity, we generated $d c p 2-N 245$ alleles harboring mutations in catalytically important residues, including those functioning as general acid (K135A) or general base (E153Q), or involved in metal-binding (E198Q) (Aglietti et al. 2013), and analyzed the effects of these mutations in YRAl premRNA decay in $d c p 2 \Delta$ and $e d c 3 \Delta d c p 2 \Delta$ cells. In contrast to the unmodified $d c p 2-N 245$ allele, the $d c p 2-N 245$ alleles harboring each of these amino acid substitutions failed to promote YRA1 pre-mRNA decay and resulted in the increased accumulation of YRA1 pre-mRNA in both $d c p 2 \Delta$ and $e d c 3 \Delta d c p 2 \Delta$ cells (Fig. 3B). These results indicate that the decreased accumulation of YRA1 pre-mRNA in edc3 $3 d c p 2 \Delta$ cells harboring the $d c p 2-N 245$ allele is indeed caused by increased activity of the decapping enzyme and suggests that the inhibitory element mainly targets the functions of the Dcp2 catalytic domain. In addition to YRA1 pre-mRNA, the $d c p 2-N 245$ allele also targets the ade2-1 mRNA, an NMD substrate. As shown in Figure $3 \mathrm{~B}, d c p 2 \Delta$ and $e d c 3 \Delta d c p 2 \Delta$ cells harboring the $d c p 2-N 245$ allele accumulated low levels of ade2-1 mRNA, but cells harboring the dcp2-N245-K135A, E153Q, or E198Q alleles all accumulated high levels of ade2-1 mRNA. Together, these results indicate that elimination of the Dcp2 inhibitory element results in constitutive activation of the decapping enzyme and that the activated enzyme appears to target substrates of both the Edc3 and NMD pathways in yeast cells.

Since deleting the putative cis-inhibitory element resulted in constitutively active Dcp2 activity for YRA1 pre-mRNA, we determined whether the constitutively active $d c p 2$ alleles depended on additional decapping regulators. We generated edc $3 \Delta d c p 2 \Delta$ cells harboring deletions of $D C P 1, U P F 1$, $D H H 1, P A T 1$, or LSM1 and analyzed the activity of the different $d c p 2$ alleles in the resulting cells. Deleting $D C P 1$ eliminated the constitutive decay activity of the $d c p 2-N 300$ and N245 alleles for YRA1 pre-mRNA (Fig. 3A), whereas deleting UPF1, DHH1, PAT1, or LSM1 had no significant effects (Supplemental Fig. S5A). These results indicate that the constitutively active $d c p 2$ alleles require Dcp1, but are independent of other decapping activators for decapping activity. Consistent with the requirement for Dcp1, the constitutively active $d c p 2$ alleles also require their N-terminal Dcp1-interacting region for mRNA decay activity (Supplemental Fig. S5B). 
Downloaded from rnajournal.cshlp.org on April 26, 2023 - Published by Cold Spring Harbor Laboratory Press
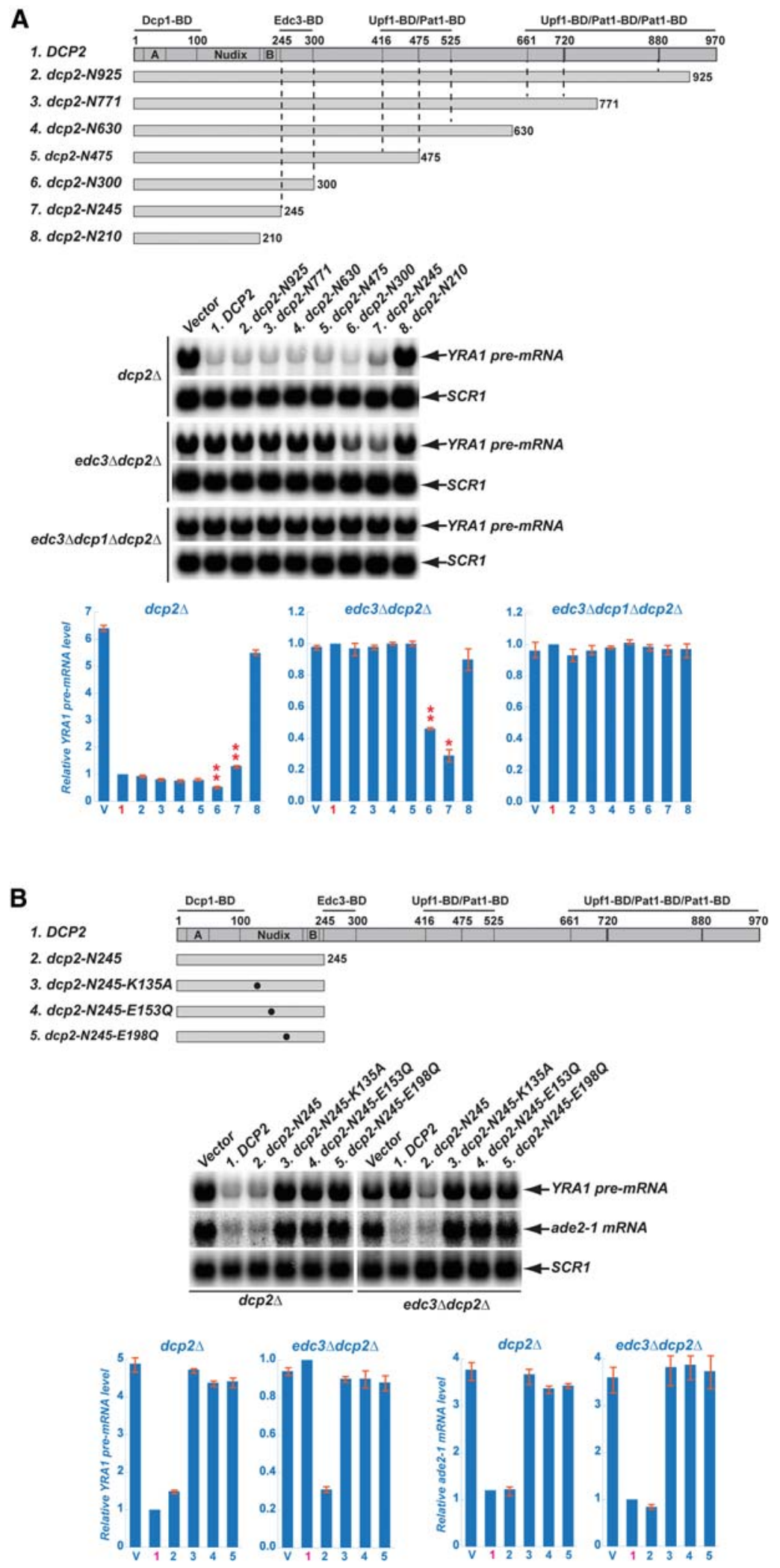

FIGURE 3. The Dcp2 C-terminal domain harbors an inhibitory element that targets the Dcp2 catalytic domain and subjects the decapping enzyme to negative regulation. (A) Northern analysis of the consequences of incremental deletions from the C-terminus of Dcp2 on YRA1 pre-mRNA decay in $d c p 2 \Delta$ and edc3 $\Delta d c p 2 \Delta$ cells. (B) Northern analysis of the effects of amino acid substitutions in the catalytically important residues on $d c p 2-N 245$ decay activity in $d c p 2 \Delta$ and edc3 $3 d c p 2 \Delta$ cells. In $A$ and $B$, schematic representations of Dcp2 domain structure and $d c p 2$ alleles used in the experiment are shown on top and the positions of the Edc3, Upf1, and Pat1-binding sites are indicated by dashed lines. Black dots in the Nudix domains shown in $B$ demark positions of amino acid substitutions. Representative Northern blots were hybridized with random-primed probes specific for YRA1 premRNA, ade2-1 mRNA, and the SCR1 transcript, with the last serving as a loading control. The relative levels of YRA1 pre-mRNA or ade2-1 mRNA derived from the Northern blots are depicted as bar graphs at the bottom of each panel. The data were the average of at least three independent experiments and were normalized to the respective cells harboring the wild-type DCP2 allele (labeled 1 in red). The red error bars indicate standard deviations. The red stars indicate the $P$-values from paired two-tailed Student's $t$-tests $\left.\left(\left[{ }^{*}\right] P<0.05,{ }^{* *}\right] P<0.01\right)$. 


\section{Activity of the Dcp2 cis-acting inhibitory element requires amino acids $350-375$}

To map the Dcp2 cis-inhibitory element more precisely, we generated additional deletions between amino acids 300 and 475 and analyzed their effects on YRA1 pre-mRNA levels in edc3 $3 d c p 2 \Delta$ cells (Fig. 4A). C-terminal deletions up to amino acid 375, including the overlapping Upf1/ Pat1-binding fragment, failed to activate Dcp2 decay activity for YRA1 pre-mRNA, but additional deletion of amino acids 350-375 was sufficient for activation. Further deletions up to amino acid 245, including the Edc3-binding site, did not enhance YRA1 pre-mRNA decay. Internal deletion of amino acids 300-350 of Dcp2 was inconsequential, but internal deletion of amino acids 300-400 promoted the YRA1 premRNA decay activity (Fig. 4B). Together, these results indicate that Dcp2's cis-inhibitory activity requires amino acids 350-375, a segment located between but distinct from the Edc3- and Upf1/Pat1-binding sites (Fig. 4A). This inhibitory element is rich in both proline and phenylalanine, and is conserved in Dcp2 orthologs from yeast species closely or remotely related to Saccharomyces cerevisiae (Fig. 4C).

To test whether the increased YRA1 pre-mRNA decay activity observed with constitutively active $d c p 2$ alleles results mainly from loss of the inhibitory element, and not from increased protein expression, we generated HA-tagged versions
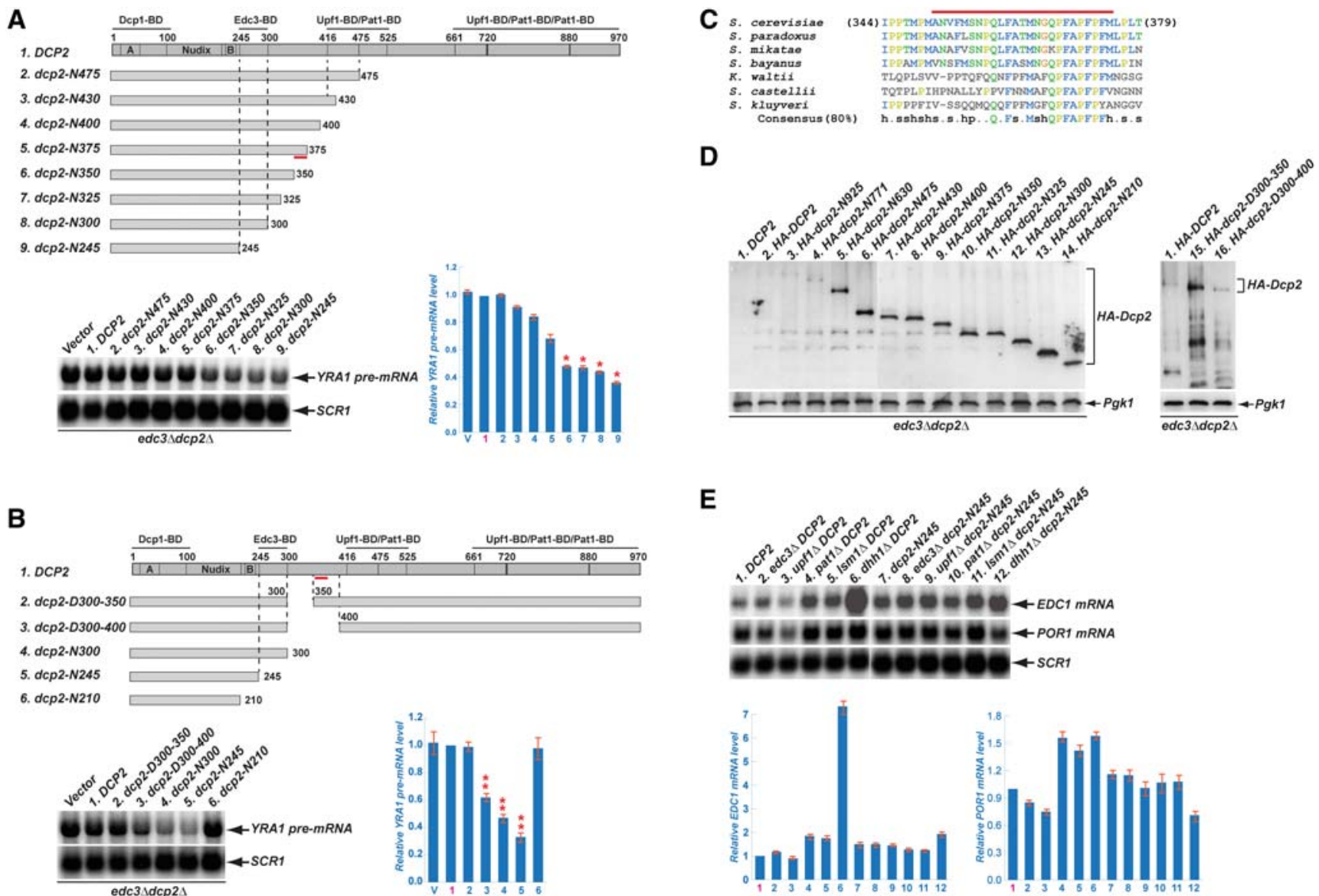

FIGURE 4. Mapping of the cis-inhibitory element in the C-terminal domain of Dcp2, and analyzing the effects of loss of this element on Dcp2 protein accumulation and mRNA decay function. (A) Northern analysis of the effects of small incremental deletions between amino acids $300-475$ of Dcp2 on YRA1 pre-mRNA decay in $e d c 3 \Delta d c p 2 \Delta$ cells. (B) Northern analysis of the effects of small internal deletions between amino acids 300-400 of Dcp2 on YRA1 pre-mRNA decay in $e d c 3 \Delta d c p 2 \Delta$ cells. $(C)$ Sequence alignment of the Dcp2 inhibitory element with conserved sequences from Dcp2 orthologs of several other yeast species. $(D)$ Western analysis of the effects of C-terminal and internal deletions on Dcp2 protein expression in $e d c 3 \Delta d c p 2 \Delta$ cells using triple-HA tagged $d c p 2$ alleles. (E) Northern analysis of the consequences of the Dcp2 C-terminal N245 deletion on EDC1 mRNA and POR1 mRNA levels in control cells or cells harboring deletions of the EDC3, UPF1, PAT1, LSM1, or DHH1 genes. In A and B, schematic representations of Dcp2 domain structure and $d c p 2$ alleles used in the experiment are shown on top and the positions of Edc3, Upf1, and Pat1-binding sites are indicated by dashed lines. The Dcp2 amino acid sequence required for cis-inhibitory activity is highlighted by a red bar. In $A, B$, and $E$, representative Northern blots were hybridized with random-primed probes specific for YRA1 pre-mRNA, EDC1 mRNA, POR1 mRNA, or the SCR1 transcript. The relative levels of YRA1 pre-mRNA, EDC1 mRNA, and POR1 mRNA derived from Northern blots are depicted as bar graphs of the corresponding panels. The data comprise the average of at least three independent experiments and were normalized to the respective cells harboring the wild-type DCP2 allele (labeled 1 in red). The red error bars indicate standard deviations. The red stars indicate the $P$-values from paired two-tailed Student's $t$-tests $\left(\left[{ }^{*}\right] P<0.05\right.$, $\left[{ }^{* *}\right] P<$ 0.01). In $D$, Western blots were probed with monoclonal antibodies against the HA epitope or Pgk1, with the latter serving as a loading control. 
of each $d c p 2$ allele analyzed above (Supplemental Fig. S6A) and examined their levels of expression and extent of activity in YRA1 pre-mRNA decay in $d c p 2 \Delta$ and edc3 $\Delta d c p 2 \Delta$ cells (Fig. 4D; Supplemental Fig. S6B,C). These experiments led to several important observations. First, each of the HA-tagged $d c p 2$ alleles behaved the same as its untagged counterpart in YRA1 pre-mRNA decay activity (compare the untagged $d c p 2$ alleles in Fig. 3A to the HA-tagged counterparts in Supplemental Fig. S6B, and data not shown), indicating that HA-tagging does not alter the function of the $d c p 2$ alleles. Second, different HA-tagged $d c p 2$ alleles exhibited different levels of protein. However, each HA-tagged $d c p 2$ allele had almost identical protein levels in $d c p 2 \Delta$ and edc3 $\Delta d c p 2 \Delta$ cells (Fig. 4D; Supplemental Fig. S6C), indicating that the presence or absence of Edc3 does not affect the expression of these $d c p 2$ alleles. Third, HA-tagged $d c p 2$ alleles harboring larger C-terminal deletions generally resulted in increased Dcp2 levels (Fig. 4D; Supplemental Fig. S6C), but these increased Dcp2 levels did not correlate with increased YRA1 pre-mRNA decay activity. For example, HA-dcp2-N430 and $H A-d c p 2-N 300$ had similar levels of Dcp2 protein (Fig. 4D; Supplemental Fig. S6C), but exhibited distinct YRA1 premRNA decay activity. The former was inert and the latter was constitutively active (Fig. 4A). Collectively, these observations indicate that the increased $Y R A 1$ pre-mRNA decay activity of the constitutively active $d c p 2$ alleles is due to loss of the inhibitory element, not to changes in the levels of protein expression. C-terminal truncations have also been shown to increase accumulation of human Dcp2 (Erickson et al. 2015). These observations indicate that in vivo levels of yeast and human Dcp 2 are both subject to regulation by protein degradation. However, whether yeast Dcp2 is also controlled by proteasome-mediated degradation is currently unknown.

Since loss of the Dcp2 cis-inhibitory activity leads to Edc3 independent decay of YRA1 pre-mRNA, this indicates that negative regulation plays a role in determining the decapping enzyme's substrate specificity. Consistent with this idea, we also found that eliminating the Dcp2 C-terminus also resulted in loss of Dhh1 control of its two substrates, EDC1 mRNA (Muhlrad and Parker 2005) and POR1 mRNA (Fig. 4E, cf. lanes 1 and 6 and lanes 7 to 12; Chang and Lee 2012).

\section{Roles of the decapping activators and their interactions with Dcp2 in decapping regulation}

In addition to harboring an inhibitory element, the Dcp2 C-terminal domain also contains a set of conserved binding sites for the decapping activators Edc3, Pat1, and Upf1 (see above). We hypothesized that interactions of these conserved binding sites with their corresponding decapping activators could control the decapping enzyme's substrate specificity, targeting, and final activation. In the following experiments, we exploited the fact that the product of the $d c p 2-N 245$ allele is a constitutively active catalytic subunit that no longer binds to Edc3, Pat1, or Upf1 to dissect the roles of the decapping ac- tivators and their interactions with Dcp2 in decapping regulation. We focused on Edc3 and Upf1. To assess the roles of Edc3:Dcp2 and Upf1:Dcp2 interactions and to determine whether Edc3 and Upf1 may have additional functions independent of their Dcp2 interactions in decapping regulation, we constructed a set of yeast strains harboring genomic wild-type DCP2, $d c p 2-N 245$, or $d c p 2-N 245$ tagged with a basic zipper R34 (Bashor et al. 2008) in EDC3, UPF1, edc3 4 , upf1 and edc3 $\Delta u p f 1 \Delta$ backgrounds and analyzed mRNA decay phenotypes for several representative transcripts of the Edc3, NMD, and general $5^{\prime}$ to $3^{\prime}$ decay pathways. As shown in Figure 5A and Supplemental Figure S7, eliminating the C-terminal domain of Dcp2 compromised both Edc3-mediated decay and NMD, causing complete stabilization of $R P S 28 B$ mRNA and partial stabilization of YRA1 premRNA, two substrates of Edc3-mediated decay (Badis et al. 2004; Dong et al. 2007), as well as modest (about 1.5-fold) but consistent stabilization of ade2-1 mRNA and CYH2 premRNA, two NMD substrates (He et al. 1993; Maderazo et al. 2003). These observations show that the constitutively active Dcp2-N245 decapping enzyme is at least partially deficient in degrading the substrates of the Edc3 and NMD pathways, probably due to loss of positive Edc3:Dcp2 and Upf1: Dcp2 interactions. One implication of these observations is that decapping of Edc3 and NMD substrates is dependent on positive regulatory mechanisms. In cells harboring either dcp2-N245 allele, eliminating Edc3 caused further stabilization of YRA1 pre-mRNA (approximately twofold) and eliminating Upf1 caused significant stabilization of ade2-1 mRNA and $\mathrm{CYH} 2$ pre-mRNA ( $>10$-fold in each case) (Fig. 5A). Since Dcp2-N245 binds neither Edc3 nor Upf1, the observed effect of deleting EDC3 or UPF1 on mRNA stability in dcp2-N245 cells most likely reflects the existence of Edc3 and Upf1 functions that are independent of Dcp 2 interactions. Furthermore, regardless of the status of EDC3 or UPF1, eliminating the C-terminal domain of Dcp2 promoted the decay of PGK1 and TCM1 mRNAs, two general mRNA decay substrates (Decker and Parker 1993; He and Jacobson 2001). This result indicates that decapping of these two general mRNAs is mostly controlled through inhibitory mechanisms that likely include the direct inhibition of the decapping enzyme by the Dcp2 inhibitory element and sequestration of the decapping enzyme by competitive Edc3:Dcp2 and Upf1:Dcp2 interactions. Consistent with this interpretation, we found that deletion of $U P F 1$ in a wild-type DCP2 background routinely led to decreased accumulation of general mRNAs (data not shown). These results suggest that Edc3 and Upf1 probably compete with Pat 1 for binding to the decapping enzyme and thus limit the available pool of decapping enzyme for general mRNAs. Together, these results indicate that Edc3:Dcp2 and Upf1: Dcp2 interactions have positive roles in targeting the decapping enzyme to specific mRNAs, but can also have negative effects on the decapping of unrelated general mRNAs. In addition, Edc3 and Upf1 both appear to have additional functions that are independent of their interactions with Dcp2. 

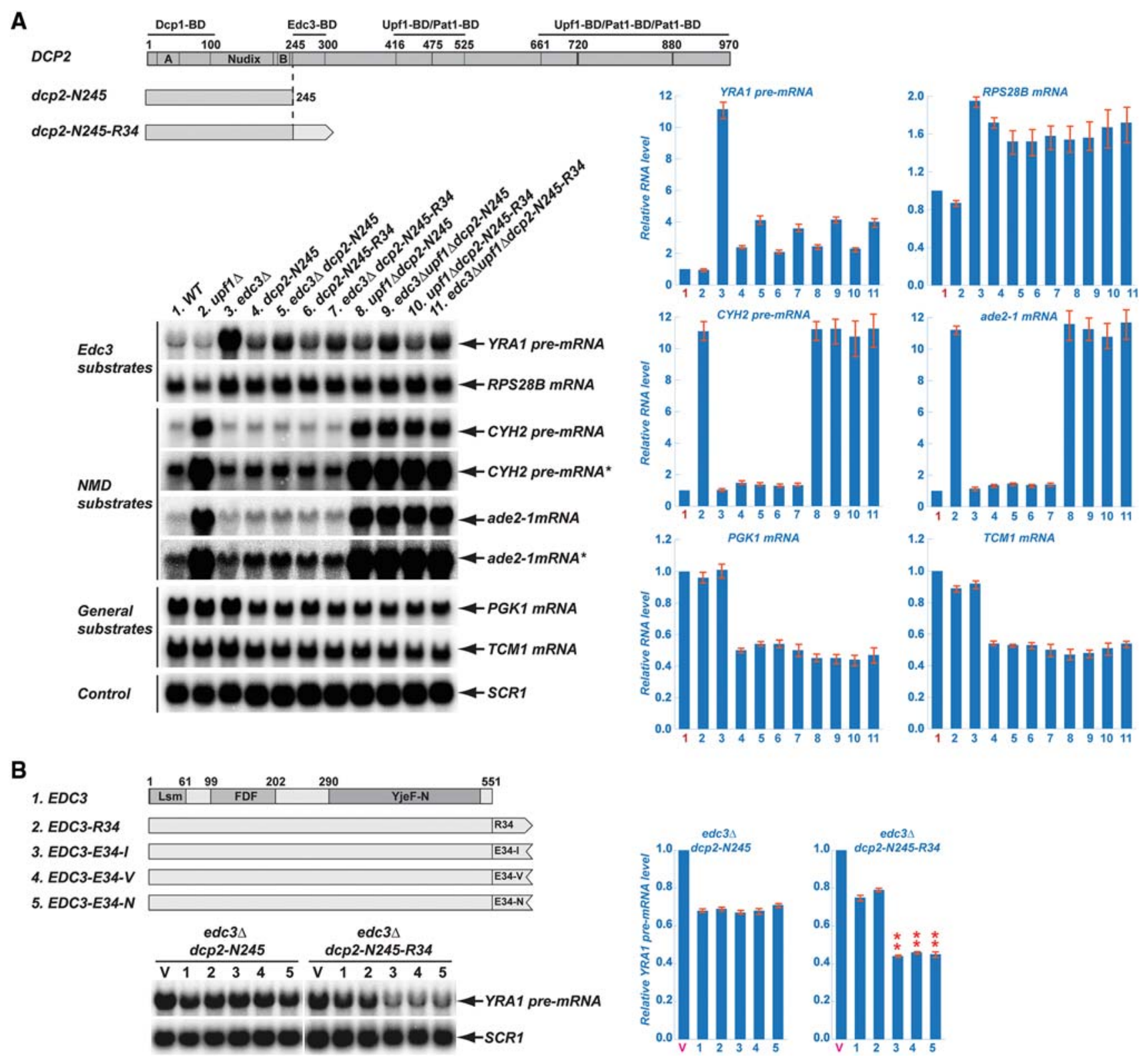

FIGURE 5. The Dcp2 C-terminal domain plays an important role in both transcript-specific and general mRNA decapping. (A) Northern analysis of the consequences of the Dcp2 C-terminal N245 deletion on Edc3-mediated mRNA decay, NMD, and general mRNA decay in yeast cells harboring either deletions of the EDC3 or UPF1 genes or the respective wild-type genes. Schematic representations of the Dcp2 domain structure and the genomic R34-tagged or untagged $d c p 2-N 245$ allele used in the experiments are shown on top. Overexposed blots for CYH2 pre-mRNA and ade2-1 mRNA are indicated by an asterisk. (B) Northern analysis of the effect of restoring Edc3 interaction with dcp2-N245 on YRA1 pre-mRNA decay. Schematic representations of the Edc3 domain structure and EDC3 alleles tagged with either a basic or acidic leucine zipper used in the experiments are shown on top. $V$ indicates the empty vector control. In $A$ and $B$, representative Northern blots were hybridized to random-primed probes specific for the indicated transcripts with the SCR 1 transcript serving as a loading control. The relative levels of each transcript derived from Northern blots are depicted in bar graphs on the right side of each panel. The data are the average of at least three independent experiments and are normalized to the cells harboring the wild-type $D C P 2$ allele $(A)$ or the empty vector $(B)$ (labeled 1 or $V$ in red, respectively). The red error bars indicate standard deviations. The red stars indicate the $P$-values from paired two-tailed Student's $t$-tests $\left(\left[{ }^{*}\right] P<0.05\right.$, $\left.\left[{ }^{* *}\right] P<0.01\right)$.

One potential role of Edc3:Dcp2 and Upf1:Dcp2 interactions is the formation of distinct decapping enzyme complexes that can target specific mRNAs. To test this possibility, we examined whether restoring interaction between Edc3 and Dcp2-N245 through artificial leucine-zippers capable of heterodimerization (Bashor et al. 2008) could promote YRA1
pre-mRNA decay. A set of EDC3 alleles with C-terminal tags of acidic E34-I, E34-V, or E34-N zippers was generated on centromere-based plasmids. These tagged EDC3 alleles were individually introduced into an edc3 $\Delta d c p 2-N 245-R 34$ strain (expressing $d c p 2-N 245$ tagged with a basic zipper) and the resulting cells were analyzed for YRA1 pre-mRNA 
levels. As controls, we also created strains harboring wildtype or R34-tagged EDC3 alleles, or the empty vector, and also introduced each EDC3 allele into an edc3 $\triangle d c p 2-$ N245 strain (expressing d $c p 2-N 245$ with no zipper). The edc3 $\Delta d c p 2-N 245-R 34$ and edc3 $\Delta d c p 2-N 245$ strains harboring the empty vector behaved identically and both showed high levels of YRA1 pre-mRNA accumulation (Fig. 5B, both lanes designated $\mathrm{V}$ ), indicating that the R34 tag does not alter the function of Dcp2-N245. When wild-type EDC3 and each of its tagged alleles were introduced into the control edc3 $\Delta d c p 2-N 245$ strain, they behaved similarly and each allele enhanced YRA1 pre-mRNA decay slightly relative to the empty vector control (Fig. 5B, left panel, cf. lanes $1-5$ to $\mathrm{V}$ ). This decay-enhancing effect most likely reflects an Edc3 function independent of its interaction with the decapping enzyme (see above). When wild-type EDC3 and each of its tagged alleles were introduced into the edc3 $\Delta d c p 2-N 245-R 34$ strain, they yielded distinct YRA1 pre-mRNA decay phenotypes. The wild-type and R34-tagged EDC3 alleles again enhanced YRA1 pre-mRNA decay slightly whereas the E34-I, E34-V, and E34-N-tagged alleles promoted more efficient decay (Fig. 5B, right panel, lanes 3-5). These results show that coexpression of Dcp2-N245 and Edc3 tagged with pairs of leucine zippers capable of heterodimerization promotes YRA1 premRNA decay. Although E34-I, E34-V, or E34-N bind R34 with different affinities in vitro $\left(K_{d}=6.1,421\right.$, and $810 \mathrm{nM}$, respectively) (Bashor et al. 2008), EDC3 alleles tagged with E34-I, E34-V, or E34-N reduced YRA1 pre-mRNA levels to similar extents, indicating that Edc3 interaction with Dcp2 readily targets the decapping enzyme to YRA1 pre-mRNA.

In addition to targeting specific transcripts, our genetic experiment in Figure 3A revealed that Edc3:Dcp2 interaction also plays an important role in decapping enzyme activation. We observed that $d c p 2$ alleles harboring the inhibitory element were completely deficient in YRA1 pre-mRNA decay in the absence of Edc3. However, each of these alleles promoted efficient YRA1 pre-mRNA degradation in the presence of Edc3. Significantly, $d c p 2$ alleles lacking the inhibitory element promoted YRA1 pre-mRNA decay even in the absence of Edc3 (Fig. 3A). These results indicate that the presence of the inhibitory element in Dcp2 imposes a strict functional requirement for Edc3 and that elimination of the inhibitory element in Dcp2 can bypass the Edc3 function for YRA1 pre-mRNA decapping. These observations argue that Edc3: Dcp2 interaction functions at least in part to counteract the inhibitory activity imposed on Dcp2, a step required for decapping activation.

\section{DISCUSSION}

\section{The yeast decapping enzyme is subject to both negative and positive regulation}

In contrast to current models that are largely based on in vitro experimentation (Floor et al. 2010; Nissan et al. 2010; Borja et al. 2011), the in vivo studies reported here reveal that the yeast decapping enzyme is subject to both negative and positive regulation. Using a combination of two-hybrid assays, deletion alleles, point mutants, and quantitative assessments of mRNA decay activity, we found that the long-neglected large C-terminal domain of Dcp2 contains two key types of regulatory elements: (i) a negative regulatory element that inhibits decapping enzyme activity and (ii) a set of positive regulatory elements that recruit the specific decapping activators Edc3, Pat1, and Upf1. Although the Dcp2 sites through which they appear to act are novel, positive regulation of the decapping enzyme by specific decapping activators is largely expected (Parker 2012). However, negative regulation of the decapping enzyme by a cis-inhibitory element in Dcp2 is surprising, and undoubtedly significant, as elimination of the inhibitory element results in constitutive activation of the decapping enzyme and loss of the enzyme's specificity for substrate mRNAs. Negative regulation of the decapping enzyme by an inhibitory element could serve two important purposes for eukaryotic cells. One would be the avoidance of accidental or unregulated mRNA decapping and the other would enable temporal control of decapping enzyme activation.

Positive regulation of the decapping enzyme appears to be mediated by the binding of specific decapping activators to the Dcp2 C-terminal domain and the likely formation of distinct complexes that control the decapping enzyme's substrate specificity and final activation (see below). Positive regulation of the yeast decapping enzyme by several decapping activators has been documented in many previous in vitro studies (Harigaya et al. 2010; Nissan et al. 2010; Fromm et al. 2012), but those results differ substantially from the in vivo experiments reported here. These differences are not surprising. The in vitro experiments, including structural, enzymatic, and protein-binding analyses (Steiger et al. 2003; Decker et al. 2007; Deshmukh et al. 2008; She et al. 2008; Floor et al. 2010; Harigaya et al. 2010; Nissan et al. 2010; Borja et al. 2011) used either of two N-terminal Dcp2 fragments (Dcp2-N245 or Dcp2-N300), both of which we have now shown to lack the inhibitory element and most or all of the binding sites for Edc3, Pat1, and Upf1, and to be constitutively active in mRNA decapping in vivo (Fig. 3A). Further, the activity of these two constitutively active forms of Dcp2 is independent of the functions of almost all characterized decapping activators, including Edc3, Pat1, Dhh1, Lsm1, and Upf1 (Supplemental Fig. S5).

\section{Regulatory functions of yeast decapping activators}

Our experiments reveal that the decapping activators Edc3, Pat1, and Upf1 bind to a set of distinct conserved regulatory elements in the C-terminal domain of Dcp2 and suggest at least two key functions for these interactions: control of the decapping enzyme's substrate specificity and triggering of enzyme activation. The latter point is best illustrated by Edc3- 
mediated decay of YRA1 pre-mRNA. Figures $3 \mathrm{~A}$ and $4 \mathrm{~A}$ demonstrate that when the inhibitory element is present in Dcp2 the decay of YRA1 pre-mRNA has a strict requirement for Edc3. In contrast, when the inhibitory element is deleted, YRA1 pre-mRNA decay can bypass its requirement for Edc3. This implies that the binding of Edc3 to Dcp2 alleviates the inhibitory activity otherwise imposed on Dcp2, thus promoting decapping of the Edc3 substrate, YRA1 pre-mRNA.

Edc3, Pat1, and Upf1 each appear to bind directly to their respective substrate mRNAs (Chowdhury et al. 2007; Johansson et al. 2007; He et al. 2014). These results, and our observations that the same proteins also bind to distinct sites in Dcp2, strongly suggest that these decapping activators directly control the targeting or substrate specificity of the decapping enzyme. Further, the binding of Edc3, Pat1, and Upf1 to the Dcp2 C-terminal domain most likely leads to the formation of distinct decapping complexes instead of a single large complex encompassing most activators, as suggested earlier (Nissan et al. 2010; Swisher and Parker 2011). Support for this conclusion follows from three observations. First, the Edc3, Pat1, and Upf1-binding sites in the Dcp2 Cterminal domain are close to each other (Fig. 1A,B), suggesting that the binding of these factors to Dcp2 may well be mutually exclusive. Second, the effects of deleting EDC3, PAT1, or UPF1 are highly specific and each of these gene deletions selectively inhibits the decay of mRNAs of a specific decay pathway, but not the unrelated decay pathways (Fig. 5A). Third, although deletion of EDC3, PAT1, or UPF1 each inhibits the activity of a specific decay pathway, we observed that deletion of UPF1 also has indirect effects on apparently unrelated mRNA decay pathways and consistently promotes the degradation of the substrates of the Edc3 or general decay pathways (Fig. 5A; Supplemental Fig. S7), i.e., elimination of the factor controlling one pathway may make additional Dcp2 available for another pathway. In turn, this implies that in addition to providing the decapping enzyme with unique substrate or targeting specificity, the formation of distinct decapping enzyme complexes by Edc3, Pat1, and Upf1 may also allow dynamic regulation between different decapping-dependent decay pathways in vivo in response to changes in development, growth, or stress signals.

In addition to roles in targeting and activating the decapping enzyme, Edc3 and Upf1 both appear to have additional functions in mRNA decapping that are independent of their interactions with Dcp2. A key observation is that in yeast cells harboring the $d c p 2-N 245$ allele, deletion of EDC3 or UPF1 resulted in significant stabilization (two- to 10-fold) of the respective substrate mRNAs (Fig. 5A). Since the $d c p 2-N 245$ allele encodes a constitutively active decapping enzyme that no longer binds to Edc3 or Upf1, these effects of EDC3 or UPF1 deletion in $d c p 2-N 245$ cells must result from loss of Edc3 or Upf1 functions that occur independently of Dcp2 interactions. One possibility is that Edc3 and Upf1 both have functions in mRNA decapping that are upstream of the formation of a decapping complex or activation of the decapping enzyme, such as repressing mRNA translation, or tagging or remodeling specific mRNPs (Coller and Parker 2005; Franks et al. 2010; Ghosh et al. 2010). The occurrence of functions independent of Dcp2 interaction, and the ability of these factors to bind directly to their substrate mRNAs (Chowdhury et al. 2007; Johansson et al. 2007; He et al. 2014), suggests that the assembly of the active decapping complexes most likely occurs on mRNAs and that Edc3 and Upf1 function in recruiting the decapping enzyme to their targeted mRNAs. Since both Edc3 and Upf1 self-associate and the self-association of these factors appears to be critical for their respective decay activities (He et al. 2013, 2014), it is possible that the presumptive upstream functions for Edc3 and Upf1 are carried out by distinct Edc3 or Upf1 molecules other than those in complexes with the decapping enzyme. In this scenario, the decapping enzyme would be recruited to the respective decay substrates by either Edc3 or Upf1 dimerization.

\section{Roles of negative and positive regulation in mRNA decapping in vivo}

Although deleting the entire C-terminal domain of Dcp2 would in principle eliminate both the negative and positive regulation proposed here, the actual consequences of that deletion did not have a uniform effect on the decapping of different transcripts, including those degraded by the same decay pathway (Fig. 5A). For example, deletion of the entire Dcp2 C-terminal domain led to complete stabilization of one Edc3 substrate, RPS28B mRNA, but only partial stabilization of another Edc3 substrate, YRA1 pre-mRNA (Fig. 5A). Concurrently, the same deletion yielded only marginal stabilization of two NMD substrates, $C Y H 2$ pre-mRNA and ade2-1 mRNA. Since elimination of the entire Dcp2 C-terminal domain resulted in constitutively active decapping enzyme, the different consequences of this deletion on decay of the Edc3 and NMD substrates may reflect distinct requirements or sensitivities of these transcripts for positive regulation. Decapping of RPS28B mRNA may require Edc3:Dcp2 interaction for both enzyme recruitment and activation whereas decapping of YRA1 pre-mRNA may require Edc3: Dcp2 interaction mostly for enzyme activation, but be less critical for the enzyme's recruitment because YRA1 premRNA is translationally repressed (Dong et al. 2010). In support of this argument, restoring the interactions between constitutively active dcp2-N245 and Edc3 only had a modest effect on YRA1 pre-mRNA decay, accelerating the decay of this transcript about twofold (Fig. 5B). The same may be true for decapping of nonsense-containing mRNAs. Such NMD substrates may be principally dependent on Upf1: Dcp2 interaction for enzyme activation, but less so for enzyme recruitment as recognition of NMD substrates also leads to translational repression (Muhlrad and Parker 1999). In addition, since deletion of UPF1 in dcp2-N245 cells still resulted in substantial stabilization of NMD substrates (Fig. 5A), Upf1- 
mediated decapping enzyme activation and recruitment probably do not control a rate-limiting step for NMD, i.e., Upf1's major function in NMD may occur earlier and involve remodeling of terminating mRNPs. Complicating the interpretation of the consequences of Dcp2 C-terminal deletion on the decay of individual transcripts is the fact that the levels of any mRNA reflect the net outcome of eliminating both the negative and positive regulation. Because loss of negative regulation results in decapping enzyme activation, any loss of positive regulation is likely to be offset to some extent by a gain of function from the loss of negative regulation. Thus, for example, the partial effect of Dcp2 C-terminal domain deletion on YRA1 premRNA levels and the marginal effect of Dcp2 C-terminal domain deletion on CYH2 pre-mRNA and ade2-1 mRNA levels are likely to be underestimates of the role of positive regulation in the decapping of these transcripts. Future analyses of small deletions or point mutations in each of these positive elements that specifically disrupt Dcp2 interactions with Edc3, Upf1, and Pat1 (or combinations of such mutations) are required to further define the roles of the positive elements in decapping regulation of different mRNAs.

Constitutive activation of the decapping enzyme caused by eliminating the Dcp2 C-terminal domain also promoted the degradation of two general transcripts, the PGK1 and TCM1 mRNAs (Fig. 5A). This result indicates that constitutively active Dcp2 also targets general mRNAs and that, in contrast to the Edc3 and NMD substrates, decapping of general mRNAs is in large part controlled through negative regulatory mechanisms. These may include direct inhibition of decapping enzyme activity by the Dcp2 inhibitory element and/or sequestration of the decapping enzyme through the formation of distinct and competing decapping complexes. Consistent with the latter possibility, we observed that, in wild-type DCP2 cells, deletion of UPF1 consistently caused decreased accumulation of general mRNAs (Supplemental Fig. S7 and data not shown). We also found that constitutively active Dcp2-N245 also targets two decapping substrate mRNAs regulated by decapping activator Dhh1 (Fig. 4E). In short, our results indicate that in vivo the decapping enzyme is controlled through both negative and positive regulatory elements.

\section{A new model for decapping enzyme regulation}

Collectively, our data reveal a pivotal role for the Dcp2 C-terminal domain in mRNA decapping and lead to a new model for decapping enzyme regulation in vivo (Fig. 6). In this model, the Dcp2 C-terminal domain contains both negative and positive regulatory elements. The negative element is thought to inhibit the enzymatic or substrate-binding activity of the decapping enzyme and promote downstream functions of the positive elements. The positive elements promote the binding of specific decapping activators (such as Edc3, Pat1, and Upf1) to Dcp2. In turn, binding of the decapping activators to the positive elements is thought to alleviate the inhibitory activity imposed on Dcp2 by the negative element and promote the formation of distinct decapping complexes. These decapping complexes possess unique substrate specificities and target specific mRNAs. Decapping activation is thus coupled to both the assembly of decapping complexes and substrate recognition by these complexes, a regulatory mechanism reminiscent of protein phosphatase regulation (Shi 2009). Much like protein phosphatases, a single decapping enzyme targets a variety of substrate mRNAs and uses stepwise assembly of decapping complexes to control both substrate specificity and enzymatic activation.

\section{MATERIALS AND METHODS}

\section{General methods}

Preparation of standard yeast media and methods of cell culture were as described previously (Guthrie and Fink 1991). Transformation of yeast was done by the high-efficiency method (Schiestl and Gietz 1989), and DNA manipulations were performed by standard techniques (Sambrook et al. 1989). All PCR amplifications were performed with Taq DNA polymerase and confirmed by DNA sequencing.

\section{Yeast strains}

All strains used in this study are listed in Supplemental Table S1. Strains containing complete deletions of DCP1, DCP2, EDC3, PAT1, LSM1, DHH1, and UPF1 were constructed by gene 
replacement (Guthrie and Fink 1991), using DNA fragments harboring the corresponding null alleles. All null alleles were generated from plasmid DNA by restriction enzyme digestion except for the edc3::KanMX6 allele. The edc3::KanMX6 allele was generated by PCR using the oligonucleotide pair EDC3-DSK-F and EDC3$D S K-R$ (Supplemental Table S3). Strains containing a 725-amino acid C-terminal deletion of DCP2 were also constructed by gene replacement, using a DNA fragment harboring either the $d c p 2-N 245::$ KanMX6 or the dcp2-N245-R34::KanMX6 allele. Each genomic DNA deletion or integration was confirmed by PCR analysis. Deletion of DCP1 or DCP2 resulted in lethality in the GGY1::171 background, but slow growth in the HFY114 background. Accordingly, all genetic experiments in this study used strains derived from HFY114.

\section{Plasmids}

All plasmids used in this study are listed in Supplemental Table S2. Plasmids harboring the upf1::HIS3, dcp2::HIS3, dcp2-N245::Kan $M X 6$, and edc3::URA3 alleles, or containing UPF1 fragments fused to either the GAL4 DNA-binding domain (DB) or activation domain (AD), or containing EDC3 fragments fused to GAL4-AD were described previously (He et al. 1997, 2013, 2014). Details of plasmid construction are described in Supplemental Information.

\section{Oligonucleotides}

The oligonucleotides used in this study were obtained from Eurofins MWG/Operon, Inc., and are listed in Supplemental Table S3.

\section{Yeast two-hybrid interaction assay}

Two-hybrid assays utilized previously described procedures (Fields and Song 1989). All the two-hybrid interactions were initially assayed in the GGY1::171 tester strain. In each case, a GAL4(DB) fusion construct was cotransformed with a GAL4(AD) construct into the tester strain. Transformants were incubated for 3-5 d at $30^{\circ} \mathrm{C}$ until colonies were fairly large and then replicated on X-Galcontaining plates. The color phenotypes of two independent transformants from each interaction assay were presented in the figures. To assess the potential for self-activation, each of the GAL4(DB) fusions was also cotransformed with a $G A L 4(A D)$ empty vector into the tester strain and assayed in parallel with constructs testing for interaction (Supplemental Fig. S2C). In experiments where interactions were tested in both $D B$ and $A D$ orientations (see Supplemental Fig. S1A), success in either orientation was deemed evidence for an interaction. To assess the potential for molecular interactions that were bridged, we also carried out most two-hybrid analyses in tester strains harboring complete deletions of EDC3, PAT1, DHH1, or $U P F 1$, or a 725-amino acid C-terminal truncation of DCP2. In our two-hybrid analyses, we noticed that Pat1 and Upf1 interacted with N-terminally truncated Dcp2 fragments but failed to interact with full-length Dcp2 (Supplemental Fig. S1A). One possible explanation for this observation is that in vivo full-length Dcp2 may only interact with Pat 1 and Upf1 when it is in a specific conformation and most likely in a complex with Dcp1 and Dcp1 is a limiting factor for this complex formation. This explanation is supported by observations that Upf1 interacted with Dcp1 and Upf1::Dcp1 interaction is bridged though Dcp2 (Supplemental Fig. S1A,B). Coexpression of several combinations of GAL4-DB and GAL4-AD fusions resulted in lethality in the dhh1 $\Delta$ background (Supplemental Fig. S1B). The underlying mechanism for the lethality is currently unclear, but is probably related to Dhh1 function in mRNA decapping.

\section{RNA analysis}

Cells were grown in either YEPD (Figs. 4E, 5A; Supplemental Fig. $\mathrm{S} 7$ ), or synthetic complete (SC) medium lacking leucine (Figs. 3A, B, 4A,B, 5B; Supplemental Figs. S5, S6B,C). In each case, cells (15 $\mathrm{mL}$ ) were grown at $30^{\circ} \mathrm{C}$ to an $\mathrm{OD}_{600}$ of 0.7 and harvested by centrifugation. Cell pellets were frozen on dry ice and then stored at $-80^{\circ} \mathrm{C}$ until RNA isolation. The procedures for RNA isolation and Northern blotting were as previously described (He and Jacobson 1995). Transcript-specific signals on Northern blots were determined with a FUJI BAS-2500 analyzer. Each experiment was repeated independently at least three times and data are presented as mean \pm SD. Statistical significance of the data was assessed by paired twotailed Student's $t$-tests.

\section{Analysis of protein levels}

Preparation of whole-cell extracts and Western blotting procedures were as described previously (Dong et al. 2007). The Western blots in Figure 4D and Supplemental Figure S6C were probed with monoclonal antibodies against the HA epitope (H6908, Sigma) or against Pgk1 (22C5-D8, Molecular Probes), with the latter polypeptide serving as a loading control. Proteins were detected using ECL Western blotting detection reagents and Kodak BioMax film.

\section{Protein motif analysis}

Amino acid sequences of fungal Dcp2 orthologs were downloaded from the Saccharomyces Genome Database (SGD) (http://www. yeastgenome.org). Sequence similarity between different Upf1 or Pat1-interacting segments and sequence conservation of the Dcp1, Edc3, Pat1, and Upf1-binding domains or motifs and the Dcp2 cis-inhibitory element among the Dcp2 orthologs were carried out with the multiple sequence alignment software Clustal Omega hosted on a web server (http://www.ebi.ac.uk/Tools/msa/).

\section{SUPPLEMENTAL MATERIAL}

Supplemental material is available for this article.

\section{ACKNOWLEDGMENTS}

This study was supported by National Institutes of Health grant R37GM27757 to A.J. We thank Dr. Wendell Lim (University of California at San Francisco) for the artificial leucine zipper constructs, and members of the Jacobson laboratory for helpful comments on the manuscript.

Author contributions: F.H. designed and performed the experiments. F.H. and A.J. analyzed data and prepared the manuscript.

Received April 30, 2015; accepted June 8, 2015. 


\section{REFERENCES}

Aglietti RA, Floor SN, McClendon CL, Jacobson MP, Gross JD. 2013. Active site conformational dynamics are coupled to catalysis in the mRNA decapping enzyme Dcp2. Structure 21: 1571-1580.

Badis G, Saveanu C, Fromont-Racine M, Jacquier A. 2004. Targeted mRNA degradation by deadenylation-independent decapping. Mol Cell 15: 5-15.

Bashor CJ, Helman NC, Yan S, Lim WA. 2008. Using engineered scaffold interactions to reshape MAP kinase pathway signaling dynamics. Science 319: 1539-1543.

Borja MS, Piotukh K, Freund C, Gross JD. 2011. Dcp1 links coactivators of mRNA decapping to Dcp2 by proline recognition. RNA 17: 278-290.

Chang LC, Lee FJ. 2012. The RNA helicase Dhhlp cooperates with Rbplp to promote porin mRNA decay via its non-conserved C-terminal domain. Nucleic Acids Res 40: 1331-1344.

Chowdhury A, Mukhopadhyay J, Tharun S. 2007. The decapping activator Lsm1p-7p-Patlp complex has the intrinsic ability to distinguish between oligoadenylated and polyadenylated RNAs. RNA 13: 998-1016.

Coller J, Parker R. 2005. General translational repression by activators of mRNA decapping. Cell 122: 875-886.

Decker CJ, Parker R. 1993. A turnover pathway for both stable and unstable mRNAs in yeast: evidence for a requirement for deadenylation. Genes Dev 7: 1632-1643.

Decker CJ, Teixeira D, Parker R. 2007. Edc3p and a glutamine/asparagine-rich domain of Lsm $4 p$ function in processing body assembly in Saccharomyces cerevisiae. J Cell Biol 179: 437-449.

Deshmukh MV, Jones BN, Quang-Dang DU, Flinders J, Floor SN, Kim C, Jemielity J, Kalek M, Darzynkiewicz E, Gross JD. 2008. mRNA decapping is promoted by an RNA-binding channel in Dcp2. Mol Cell 29: 324-336.

Dong S, Li C, Zenklusen D, Singer RH, Jacobson A, He F. 2007. YRA1 autoregulation requires nuclear export and cytoplasmic Edc3p-mediated degradation of its pre-mRNA. Mol Cell 25: 559-573.

Dong S, Jacobson A, He F. 2010. Degradation of YRA1 pre-mRNA in the cytoplasm requires translational repression, multiple modular intronic elements, Edc3p, and Mex67p. PLoS Biol 8: e1000360.

Dunckley T, Parker R. 1999. The DCP2 protein is required for mRNA decapping in Saccharomyces cerevisiae and contains a functional MutT motif. EMBO J 18: 5411-5422.

Dutko JA, Kenny AE, Gamache ER, Curcio MJ. 2010. 5' to 3' mRNA decay factors colocalize with Tyl gag and human APOBEC3G and promote Ty1 retrotransposition. J Virol 84: 5052-5066.

Erickson SL, Corpuz EO, Maloy JP, Fillman C, Webb K, Bennett EJ, Lykke-Andersen J. 2015. Competition between decapping complex formation and ubiquitin-mediated proteasomal degradation controls human Dcp2 decapping activity. Mol Cell Biol 35: 2144-2153.

Fields S, Song O. 1989. A novel genetic system to detect protein-protein interactions. Nature 340: 245-246.

Floor SN, Jones BN, Hernandez GA, Gross JD. 2010. A split active site couples cap recognition by Dcp2 to activation. Nat Struct Mol Biol 17: 1096-1101.

Franks TM, Singh G, Lykke-Andersen J. 2010. Upf1 ATPase-dependent mRNP disassembly is required for completion of nonsense-mediated mRNA decay. Cell 143: 938-950.

Fromm SA, Truffault V, Kamenz J, Braun JE, Hoffmann NA, Izaurralde E, Sprangers R. 2012. The structural basis of Edc3- and Scd6-mediated activation of the Dcp1:Dcp2 mRNA decapping complex. EMBO J 31: 279-290.

Ghosh S, Ganesan R, Amrani N, Jacobson A. 2010. Translational competence of ribosomes released from a premature termination codon is modulated by NMD factors. RNA 16: 1832-1847.

Guthrie C, Fink GR. 1991. Methods in enzymology: molecular biology of Saccharomyces cerevisiae, Vol. 194. Academic Press, NY.
Harigaya Y, Jones BN, Muhlrad D, Gross JD, Parker R. 2010. Identification and analysis of the interaction between Edc3 and Dcp2 in Saccharomyces cerevisiae. Mol Cell Biol 30: 1446-1456.

$\mathrm{He}$ F, Jacobson A. 1995. Identification of a novel component of the nonsense-mediated mRNA decay pathway by use of an interacting protein screen. Genes Dev 9: 437-454.

He F, Jacobson A. 2001. Upf1p, Nmd2p, and Upf3p regulate the decapping and exonucleolytic degradation of both nonsense-containing mRNAs and wild-type mRNAs. Mol Cell Biol 21: 1515-1530.

He F, Peltz SW, Donahue JL, Rosbash M, Jacobson A. 1993. Stabilization and ribosome association of unspliced pre-mRNAs in a yeast $u p f 1$ mutant. Proc Natl Acad Sci 90: 7034-7038.

He F, Brown AH, Jacobson A. 1997. Upf1p, Nmd2p, and Upf3p are interacting components of the yeast nonsense-mediated mRNA decay pathway. Mol Cell Biol 17: 1580-1594.

He F, Ganesan R, Jacobson A. 2013. Intra- and intermolecular regulatory interactions in Upf1, the RNA helicase central to nonsense-mediated mRNA decay in yeast. Mol Cell Biol 33: 4672-4684.

He F, Li C, Roy B, Jacobson A. 2014. Yeast Edc3 targets RPS28B mRNA for decapping by binding to a $3^{\prime}$-UTR decay-inducing regulatory element. Mol Cell Biol. 34: 1438-1451.

Hilgers V, Teixeira D, Parker R. 2006. Translation-independent inhibition of mRNA deadenylation during stress in Saccharomyces cerevisiae. RNA 12: 1835-1845.

Hillebrand J, Pan K, Kokaram A, Barbee S, Parker R, Ramaswami M. 2010. The Me31B DEAD-Box helicase localizes to postsynaptic foci and regulates expression of a CaMKII reporter mRNA in dendrites of Drosophila olfactory projection neurons. Front Neural Circuits 4: 121.

Hopkins KC, McLane LM, Maqbool T, Panda D, Gordesky-Gold B, Cherry S. 2013. A genome-wide RNAi screen reveals that mRNA decapping restricts bunyaviral replication by limiting the pools of Dcp2-accessible targets for cap-snatching. Genes Dev 27: 1511-1525.

Johansson MJ, He F, Spatrick P, Li C, Jacobson A. 2007. Association of yeast Upf1p with direct substrates of the NMD pathway. Proc Natl Acad Sci 104: 20872-20877.

Jonas S, Izaurralde E. 2013. The role of disordered protein regions in the assembly of decapping complexes and RNP granules. Genes Dev 27: 2628-2641.

Ma J, Flemr M, Strnad H, Svoboda P, Schultz RM. 2013. Maternally recruited DCP1A and DCP2 contribute to messenger RNA degradation during oocyte maturation and genome activation in mouse. Biol Reprod 88: 11 .

Maderazo AB, Belk JP, He F, Jacobson A. 2003. Nonsense-containing mRNAs that accumulate in the absence of a functional nonsensemediated mRNA decay pathway are destabilized rapidly upon its restitution. Mol Cell Biol 23: 842-851.

Muhlrad D, Parker R. 1999. Recognition of yeast mRNAs as "nonsense containing" leads to both inhibition of mRNA translation and mRNA degradation: implications for the control of mRNA decapping. Mol Biol Cell 10: 3971-3978.

Muhlrad D, Parker R. 2005. The yeast EDC1 mRNA undergoes deadenylation-independent decapping stimulated by Not2p, Not4p, and Not5p. EMBO J 24: 1033-1045.

Mullen TE, Marzluff WF. 2008. Degradation of histone mRNA requires oligouridylation followed by decapping and simultaneous degradation of the mRNA both $5^{\prime}$ to $3^{\prime}$ and $3^{\prime}$ to $5^{\prime}$. Genes Dev 22: 50-65.

Nissan T, Rajyaguru P, She M, Song H, Parker R. 2010. Decapping activators in Saccharomyces cerevisiae act by multiple mechanisms. Mol Cell 39: 773-783.

Parker R. 2012. RNA degradation in Saccharomyces cerevisae. Genetics 191: 671-702.

Sambrook J, Fritsch E, Maniatis T. 1989. Molecular cloning: a laboratory manual. Cold Spring Harbor Laboratory Press, Cold Spring Harbor, NY.

Schiestl RH, Gietz RD. 1989. High efficiency transformation of intact yeast cells using single stranded nucleic acids as a carrier. Curr Genet 16: 339-346. 
Schmidt MJ, West S, Norbury CJ. 2011. The human cytoplasmic RNA terminal U-transferase ZCCHC11 targets histone mRNAs for degradation. RNA 17: 39-44.

Sharif H, Conti E. 2013. Architecture of the Lsm1-7-Pat1 complex: a conserved assembly in eukaryotic mRNA turnover. Cell Rep 5: 283-291.

Sharif H, Ozgur S, Sharma K, Basquin C, Urlaub H, Conti E. 2013. Structural analysis of the yeast Dhh1-Patl complex reveals how Dhh1 engages Pat1, Edc3 and RNA in mutually exclusive interactions. Nucleic Acids Res. 41: 8377-8390.

She M, Decker CJ, Chen N, Tumati S, Parker R, Song H. 2006. Crystal structure and functional analysis of Dcp2p from Schizosaccharomyces pombe. Nat Struct Mol Biol 13: 63-70.

She M, Decker CJ, Svergun DI, Round A, Chen N, Muhlrad D, Parker R, Song H. 2008. Structural basis of Dcp2 recognition and activation by Dcp1. Mol Cell 29: 337-349.
Shi Y. 2009. Serine/threonine phosphatases: mechanism through structure. Cell 139: 468-484.

Song MG, Li Y, Kiledjian M. 2010. Multiple mRNA decapping enzymes in mammalian cells. Mol Cell 40: 423-432.

Steiger M, Carr-Schmid A, Schwartz DC, Kiledjian M, Parker R. 2003. Analysis of recombinant yeast decapping enzyme. RNA 9: 231-238.

Swisher KD, Parker R. 2011. Interactions between Upf1 and the decapping factors Edc3 and Pat1 in Saccharomyces cerevisiae. PLoS One 6: e26547.

Wu D, Muhlrad D, Bowler MW, Jiang S, Liu Z, Parker R, Song H. 2014. Lsm 2 and Lsm3 bridge the interaction of the Lsm1-7 complex with Pat1 for decapping activation. Cell Res 24: 233-246.

Xu J, Chua NH. 2012. Dehydration stress activates Arabidopsis MPK6 to signal DCP1 phosphorylation. EMBO J 31: 1975-1984.

Xu J, Yang JY, Niu QW, Chua NH. 2006. Arabidopsis DCP2, DCP1, and VARICOSE form a decapping complex required for postembryonic development. Plant Cell 18: 3386-3398. 

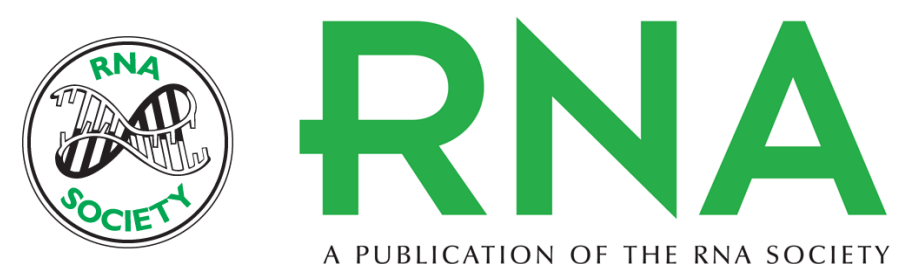

A PUBLICATION OF THE RNA SOCIETY

\section{Control of mRNA decapping by positive and negative regulatory elements in the Dcp2 C-terminal domain}

Feng $\mathrm{He}$ and Allan Jacobson

RNA 2015 21: 1633-1647 originally published online July 16, 2015

Access the most recent version at doi:10.1261/rna.052449.115

\section{Supplemental http://rnajournal.cshlp.org/content/suppl/2015/07/10/rna.052449.115.DC1 Material}

References This article cites 52 articles, 30 of which can be accessed free at: http://rnajournal.cshlp.org/content/21/9/1633.full.html\#ref-list-1

Creative This article is distributed exclusively by the RNA Society for the first 12 months after the Commons License full-issue publication date (see http://rnajournal.cshlp.org/site/misc/terms.xhtml). After 12 months, it is available under a Creative Commons License (Attribution-NonCommercial 4.0 International), as described at http://creativecommons.org/licenses/by-nc/4.0/.
Email Alerting Receive free email alerts when new articles cite this article - sign up in the box at the Service top right corner of the article or click here.

\section{|||||||| Providing Precise Solutions for your research.}

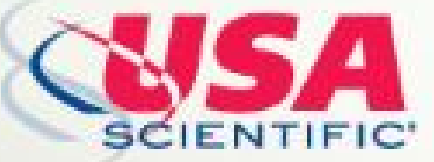

To subscribe to $R N A$ go to:

http://rnajournal.cshlp.org/subscriptions

(C) 2015 He and Jacobson; Published by Cold Spring Harbor Laboratory Press for the RNA Society 